\title{
Effect of Phosphoribosyltransferase Down-regulation on Malignant Glioma Cell Characteristics
}

\author{
MINORI KAMADA ${ }^{1,2}$, KEIICHI IKEDA $^{2}$ and YOSHINOBU MANOME ${ }^{2}$ \\ ${ }^{1}$ Division of Molecular Genetics, Core Research Facilities for Basic Science, \\ Research Center for Medical Sciences, The Jikei University School of Medicine, Tokyo, Japan; \\ ${ }^{2}$ Division of Molecular Cell Biology, Core Research Facilities for Basic Science, \\ Research Center for Medical Sciences, The Jikei University School of Medicine, Tokyo, Japan
}

\begin{abstract}
Background/Aim: Nicotinamide phosphoribosyltransferase (NAMPT) is a rate-limiting enzyme in the pathway synthesizing nicotinamide adenine dinucleotide $(N A D(+))$ from nicotinamide (NAM). Glioma tissues exhibit up-regulated NAMPT expression associated with a poor prognosis of patients. To determine if NAMPT can be a molecular therapeutic target, we investigated the effects of short hairpin RNA (shRNA)-mediated NAMPT down-regulation. Materials and Methods: We designed shRNA to NAMPT and transfected to T98G cells. The characteristics of these cells were analyzed. Results: The NAMPT shRNA-transfected cells exhibited delayed cell growth. However, there was no difference in the increase of sensitivity to temozolomide (TMZ) or X-ray irradiation between the NAMPT and scramble shRNAtransfected cells. The expression of NAMPT in the NAMPT shRNA-transfected cells increased with cell passage. Additionally, the shRNA-mediated transfection was associated with enhanced expression of quinolinic acid phosphoribosyltransferase (QPRT). Conclusion: shRNA-mediated NAMPT down-regulation may not decrease the NADt to a sufficient level to increase TMZ/radiation sensitivity.
\end{abstract}

Patients with glioblastoma (GBM), a highly malignant brain tumor, have a short life expectancy and a high rate of tumor recurrence after treatment. Also, GBMs exhibit resistance to currently-used anti-cancer therapies. The standard therapies for GBM involve surgical resection of the tumor, radiation

This article is freely accessible online.

Correspondence to: Dr. Minori Kamada, Volunteer Office, The Jikei University School of Medicine, 3-19-18 Nishishimbashi, Minatoku, Tokyo, 105-8471, Japan. Tel: +81 0334331111 Ext 5156, Fax: +810354001279, e-mail: mkamada@jikei.ac.jp

Key Words: Nicotinamide phosphoribosyltransferase, glioma, shRNA. therapy, and chemotherapy using temozolomide (TMZ), a DNA alkylating agent. However, the mean survival period and five-year survival rate of patients with GBM are 14.6 months and $9.8 \%$, respectively. Most patients with GBM exhibit tumor recurrence and most recurrent tumors are resistant to TMZ treatment. Currently, there is no effective therapy for TMZ-resistant tumors. Hence, there is an urgent need to develop a new adjuvant therapy (1).

$\mathrm{NAD}(+)$ is an essential coenzyme that mediates the redox reactions in cells and is a substrate of NAD-dependent enzymes. Cancer cells require higher $\mathrm{NAD}(+)$ levels than healthy cells, as $\mathrm{NAD}(+)$ is rapidly consumed for cancer cell growth and DNA repair (2). In mammals, $N A D(+)$ is synthesized from four precursors. One of the major $\mathrm{NAD}(+)$ synthetic pathways is the synthesis of $\mathrm{NAD}(+)$ from nicotinamide (NAM) in a two-step process. Nicotinamide phosphoribosyltransferase (NAMPT) synthesizes nicotinamide mononucleotide (NMN) by catalyzing the addition of 5-phosphoribosyl to NAM, which is the first rate-limiting step in the pathway. NMN adenylyltransferase (Nmmat) catalyzes the conversion of $\mathrm{NMN}$ to $\mathrm{NAD}(+)$. NAMPT catalyzes the rate-limiting step and hence is considered to be one of the intracellular NAD pool regulators (2).

In addition to NAM, NAD(+) is also synthesized from nicotinic acid (NA), nicotinamide riboside (NR), and tryptophan in mammals, which is catalyzed by nicotinic acid phosphoribosyltransferase (NAPRT), nicotinamide riboside kinase 1/2 (NRK1/2), and quinolinic acid phosphoribosyltransferase (QPRT), respectively (3-6).

NAMPT is the enzyme regulating the intracellular NAD pool. Hence, cancer cells exhibit a higher sensitivity to NAMPT inhibitor than healthy cells. (2) Several studies have reported that the expression of NAMPT is up-regulated in cancers, such as colon cancer, breast cancer, prostatic carcinoma, or thyroid carcinoma (7-9). In glioma, by analyzing multiple public databases, the enhanced NAMPT expression was associated with advanced tumor grade and poor overall survival in the Kaplan-Meier curve even among patients with the same tumor grade (7). 
There are several small-molecule inhibitors (SMIs) for NAMPT, such as FK866 and GMX1777. Several studies have reported that FK866 at a low concentration, which does not exert cytotoxicity, enhances the sensitivity of tumors to TMZ (8, 10). Additionally, the combination of NAMPT inhibitor and radiation therapy was reported to decrease the intracellular $\mathrm{NAD}(+)$ level and markedly delay the tumor progression of the xenografted cancer cell (11-13). In cases of glioma, however, it is improbable that the systematically administered NAMPT SMIs would pass the blood-brain barrier (BBB). In addition, gliomas rarely spread outside of central nervous system (CNS). Most glioma recurrences occur due to the remnants of tumor cells at the resection stump. Thus, a definitive localized treatment at the tumor site and surrounding areas may improve prognosis $(14,15)$.

Recent advances in gene transfer technologies have raised the hope for therapies based on gene expression modulation, such as gene editing, through direct transfer of nucleic acid to the target sites. These technologies may enable the direct modulation of protein or enzyme expression in the tumor. In this study, we hypothesized that suppressing NAMPT expression can be a potential adjuvant therapy for invasive glioma. Thus, we investigated the effect of short hairpin RNA (shRNA)-mediated NAMPT down-regulation on a glioma cell line.

\section{Materials and Methods}

Cell culture. The human T98G glioma cells and human HeLa cervical carcinoma cells were cultured in Dulbecco's minimal essential medium (DMEM) supplemented with $10 \%$ fetal bovine serum (FBS).

Preparation of retroviral vector containing shRNA targeting NAMPT. The following shRNA sequences were designed to target the NAMPT gene: 5'-CCT ACA AGG TTA CTC ACT ATA-3' (targeting nucleotides 358-378 of the NAMPT mRNA sequence, which corresponds to exon 2), 5'-GCG ATA GCT ATG ACA TTT ATA-3' (targeting nucleotides 1141-1161 of the NAMPT mRNA sequence, which corresponds to exon 7) (16), 5'-GCA TGA CAT GTG TTT CAA TAG-3' (targeting nucleotides 2916-2936 of the NAMPT mRNA sequence, which corresponds to exon 11), and 5'-GCT GCAATA GAA GCC AAA TGA3' (targeting nucleotides 3189-3209 of the NAMPT mRNA sequence, which corresponds to exon 11). These sequences were designed according to the target indicated in the databases of multiple biomaterial corporations. These sequences were cloned into the BamH I and Hind III restriction sites of the pSilencer 5.1-H1 Retro (Ambion ${ }^{\circledR}$ Thermo Fisher Scientific K.K, Tokyo, Japan) vector. In this vector, the noncoding shRNA transcription is driven by the $\mathrm{H} 1$ promoter. The sequence of the scramble shRNA was 5'-GTA CTG CTT ACG ATA CGG TCT CTTG AAC CGT ATC GTA AGC AGT ACT-3'. This is the control sequence widely applied in the vector of the particular manufacturer.

Transfection of shRNA vectors. The scramble shRNA or NAMPT shRNA-containing vectors were transfected into the T98G glioma cells using GENE PULSER II (Bio-Rad, Tokyo, Japan) at $250 \mathrm{~V}$ and $975 \mu \mathrm{F}$. The transfected cells were selected in the presence of $1.0 \mu \mathrm{g} / \mathrm{ml}$ puromycin (InvivoGen, Nacalai Tesque, Kyoto, Japan).
Immunoblotting. The transfected or wild-type cells were lysed in cold Chaps Cell Extract Buffer (50 mM Pipes/HCl (pH 6.5), 2 $\mathrm{mM}$ EDTA, $0.1 \%$ Chaps, $20 \mu \mathrm{g} / \mathrm{ml}$ leupeptin, $10 \mu \mathrm{g} / \mathrm{ml}$ pepstatin A, $10 \mu \mathrm{g} / \mathrm{ml}$ aprotinin, $5 \mathrm{mM}$ dithiothreitol (DTT), $1 \mathrm{mM}$ phenylmethylsulfonyl fluoride; Cell Signaling Technology, Danvers, MA) by subjecting the cells to 3 freeze-thaw cycles. The cell lysates were centrifuged and the supernatant was used for further analysis. The amount of protein in the supernatant was determined. Equal amounts $(5 \mu \mathrm{g})$ of proteins were resolved by sodium dodecyl sulfate-polyacrylamide gel electrophoresis (SDS-PAGE). The resolved proteins were transferred to a polyvinylidene fluoride (PVDF) membrane. The membrane was then probed with the following primary antibodies: purified rabbit polyclonal anti-NAMPT (center) (1:500; AP9010c, ABGENT, California, USA), anti-NAPRT (1:500; CL0665, AMAb90823, Atlas Antibodies), rabbit polyclonal anti-QPRT (1:250; HPA011887, Atlas Antibodies), rabbit polyclonal antiNRK1 (1:1000; NBP1-79662, Novus Biologicals, USA), monoclonal anti-MGMT (1:500; clone MT3.1, MAB16200, Chemicon, Millipore, Merck, Tokyo, Japan), mouse monoclonal anti $\beta$-actin (1:500; AC-74, A5316, Sigma-Aldrich), mouse monoclonal anti $\beta$-actin (8H10D10, 1:1,000; 3700S, Cell Signaling Technology, Danvers, MA), and rabbit polyclonal IgG anti $\beta$-actin (1:250; N-21, sc-130656, Santa Cruz Biotechnology, TX, USA). The membrane was then probed with the following secondary antibodies: goat anti-rabbit IgG-horseradish peroxidase (HRP) $(1: 1,000 ;$ SC-2004, Santa Cruz Biotechnology, Texas, USA) and ECL ${ }^{\mathrm{TM}}$ anti-mouse IgG HRP-linked $\mathrm{F}\left(\mathrm{ab}^{\prime}\right)_{2}$ fragment $(1: 1,000 ;$ NA9310V, GE Healthcare). The protein bands were visualized by enhanced chemiluminescence (ECL) (Thermo Pierce Western Blotting Substrate, Thermo Fisher Scientific, Tokyo, Japan).

Growth curve. The growth of the cell lines was assessed in 6-cm culture dishes. The cells were seeded at a density of $1.0 \times 10^{5}$ cells per dish. The cell growth was determined by viable cell counts with the method of trypan-blue dye exclusion.

Drug. Temozolomide (TMZ; T1849, LKT Laboratories, Saint Paul, MN, USA) was dissolved in the culture medium and the medium was filter-sterilized using a $0.22-\mu \mathrm{m}$ filter.

Cytotoxicity assay. The cells were seeded in 96-well sterile plates at a density of $1.25 \times 10^{3}$ cells per well. After $24 \mathrm{~h}$, the cells were incubated with serial dilutions of TMZ for $120 \mathrm{~h}$. To assess the cell viability, the cells were fixed with $3.3 \%$ glutaraldehyde for $15 \mathrm{~min}$ at room temperature and stained with $0.05 \%$ methylene blue for 15 $\mathrm{min}$. The cells were washed and the dye was eluted with $0.4 \mathrm{~N} \mathrm{HCl}$ for $15 \mathrm{~min}$. The absorbance of the mixture at $595 \mathrm{~nm}$ was measured in a microplate reader (model $680 \mathrm{XR}$, Bio-Rad, Tokyo, Japan). The sensitivity to TMZ was evaluated by calculating the half-maximal inhibitory concentration $\left(\mathrm{IC}_{50}\right)$ of the drug (17).

Colony formation assay. The wild-type, scramble shRNAtransfected and NAMPT shRNA-transfected T98G glioma cells were incubated in triplicates in $25 \mathrm{~cm}^{2}$ flasks. The cells in each flask were irradiated with 0,2 , and 4 Gy X-ray using the X-ray radiation device (MBR-1520R-3, Hitachi Engineering \& Service Co., Ltd., Yokohama, Kanagawa, Japan). The cells were trypsinized and transferred to 6-well plates (IWAKI, Shizuoka, Japan). After 1015 days, the colonies were fixed with $3.3 \%$ glutaraldehyde and 
stained with $0.05 \%$ methylene blue. The number of colonies was determined after washing.

Measuring the intracellular NADt, NADH, NAD(+), NADPt, $N A D P H$, and $N A D P(+)$ levels. The intracellular $\mathrm{NAD}(+)$ and $\mathrm{NADP}(+)$ levels were measured using the NAD/NADH Quantification Colorimetric Kit (BioVision, CA, USA) and NADP/NADPH Assay Kit-WST (Dojindo Laboratories, Kumamoto, Japan), respectively, following the manufacturer's instructions. The NADt or NADH level was calculated using the following equation:

NADt or NADH level per well $=\left[\left(\mathrm{OD}_{\text {sample }}\right) /\left(\left(\mathrm{OD}_{\text {sample }}+\right.\right.\right.$ Spike $)-$ $\left.\left(\mathrm{OD}_{\text {sample }}\right)\right) \times \mathrm{NADH}$ Spike $\left.(\mathrm{pmol})\right]$, where $\mathrm{OD}_{\text {sample }+ \text { spike }}$ is the $\mathrm{OD}$ of the sample to which a known amount of NADH was added and $\mathrm{OD}_{\text {sample }}$ is the OD of the sample to which NADH was not added.

The amount of NADt and NADH was divided by the cell number and expressed in pmol/106 cells. The $\mathrm{NAD}(+)$ concentrations were calculated by subtracting the NADH content from the NADt content. The NAD/NADH ratio is calculated as follows: (NADt $\mathrm{NADH}) / \mathrm{NADH}$. The NADPt or NADPH amount was calculated from the standard curve. The NADP(+) amount and NADP/NADPH ratio were calculated using the same method used for calculating the $\mathrm{NAD}(+)$ amount.

Quantification of RNA transcripts. Total RNA was extracted from the T98G and HeLa cells using the acid guanidium-phenol-chloroform (AGPC) method (TRIZOL ${ }^{\circledR}$ Reagent, Invitrogen, Thermo Fisher Scientific). The isolated RNA sample was treated with a recombinant RNase inhibitor (Takara Bio Inc., Shiga, Japan) and recombinant RNase-free recombinant DNase I (Takara Bio Inc.). The resulting DNA-free RNAs were reverse transcribed to cDNA using the Takara PrimeScript RT Master Mix (Takara Bio Inc., Shiga, Japan). The mRNAs of NAMPT, NAPRT, NRK, and QPRT were subjected to semi-quantitative polymerase chain reaction using SYBR ${ }^{\circledR}$ Premix Ex TaqTM II (Takara Bio Inc.) in LightCycler ${ }^{\circledR} 96$ (Roche Diagnostics). The PCR conditions were as follows: $95^{\circ} \mathrm{C}$ for $30 \mathrm{~s}$, followed by 50 cycles of $95^{\circ} \mathrm{C}$ for $5 \mathrm{~s}$ and $60^{\circ} \mathrm{C}$ for $20 \mathrm{~s}$. The primer pairs used for the analysis were as follows: For NAMPT, 5'-TCAAGAAGTACA CAGGCACCA-3' (Forward) and 5'-GTGGCAGCAACTTGTAA CCC-3' (Reverse); For NAPRT1, 5'-CATTGGCACCAGTGTGG TCA-3' (Forward) and 5'-TCCTCGGTCAGCTTCATTCG-3' (Reverse); For NRK1, 5'-CACCTCCCAAATTGCAGTGTC-3' (Forward) and 5'-TCCAGCAGGAAATGGCTGAC-3' (Reverse); For QPRT, 5'-GTCCTGCTGGACAACTTCAAGC-3' (Forward) and 5'-TGGGTCAGCATCCCCATGGAG-3' (Reverse) (18); For GAPDH, 5'-AGGAGTAAGACCCCTGGACC-3' (Forward) and 5'-ACATGGCAACTGTGAGGAGG-3' (Reverse). The specificities of the reaction were confirmed by generating the melting curve under the following conditions: $95^{\circ} \mathrm{C}$ for $1 \mathrm{~s}, 65^{\circ} \mathrm{C}$ for $15 \mathrm{~s}, 95^{\circ} \mathrm{C}, 5$ readings $/{ }^{\circ} \mathrm{C}$ following the PCR reaction.

Statistical analysis. Data were analyzed by the Student's $t$-test. Significance was determined by $p$-value under 0.05 .

\section{Results}

Preparation of the NAMPT shRNA-transfected cells and determination of MGMT expression. We comparatively evaluated the expression levels of NAMPT in the T98G cells

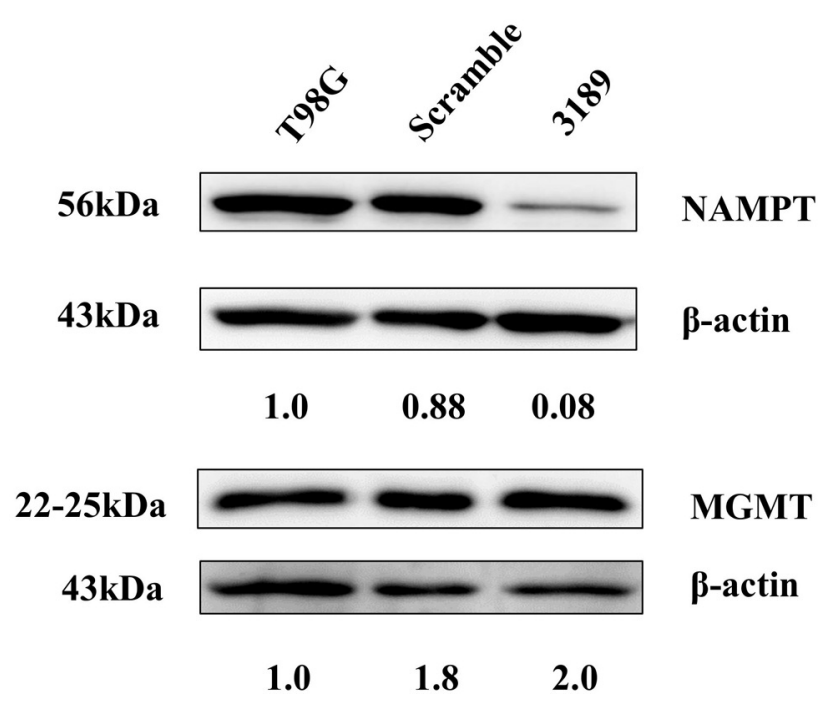

Figure 1. Western blot analysis of nicotinamide phosphoribosyltransferase (NAMPT) and $O^{6}$-methylguanine-DNA methyltransferase (MGMT) expression in the wildtype, scramble short hairpin RNA (shRNA)-transfected, and NAMPT shRNA-transfected T98G cells. The MGMT expression level in the NAMPT shRNA-transfected cells was found similar to that of the scramble shRNA-transfected cells. $\beta$-Actin was used as a loading control.

after transfection with 4 different NAMPT shRNAs. For further experiments, we used a stable cell line, which was transfected with shRNA targeting the nucleotides 3189-3209 of the NAMPT mRNA sequence, exhibiting low expression levels of NAMPT. The immunoblotting analysis revealed that the scramble shRNAtransfected cells exhibited $88 \%$ of the NAMPT protein expression, whereas the NAMPT shRNA-transfected cells exhibited $8 \%$ of the NAMPT protein expression observed in the wildtype cells (Figure 1, upper panel).

The expression of methylguanine-DNA-methyltransferase (MGMT), a DNA repair enzyme, determines the sensitivity to TMZ in glioma treatment. Several studies have reported that TMZ sensitivity increases by suppressing NAMPT expression $(8,10)$. Therefore, we investigated the protein expression level of MGMT in the NAMPT shRNAtransfected cells by immunoblotting in the same samples used for investigating NAMPT expression (Figure 1, lower panel). The NAMPT shRNA-transfected cells exhibited similar MGMT expression levels as the scramble shRNAtransfected cells.

Growth of NAMPT shRNA-transfected cells. The doubling time of the wildtype, scramble shRNA-transfected, and NAMPT shRNA-transfected cells was measured to confirm that the NAMPT shRNA-transfected cells exhibited delayed cell growth when compared to the wildtype and scramble shRNA-transfected cells. The doubling times of wildtype, 


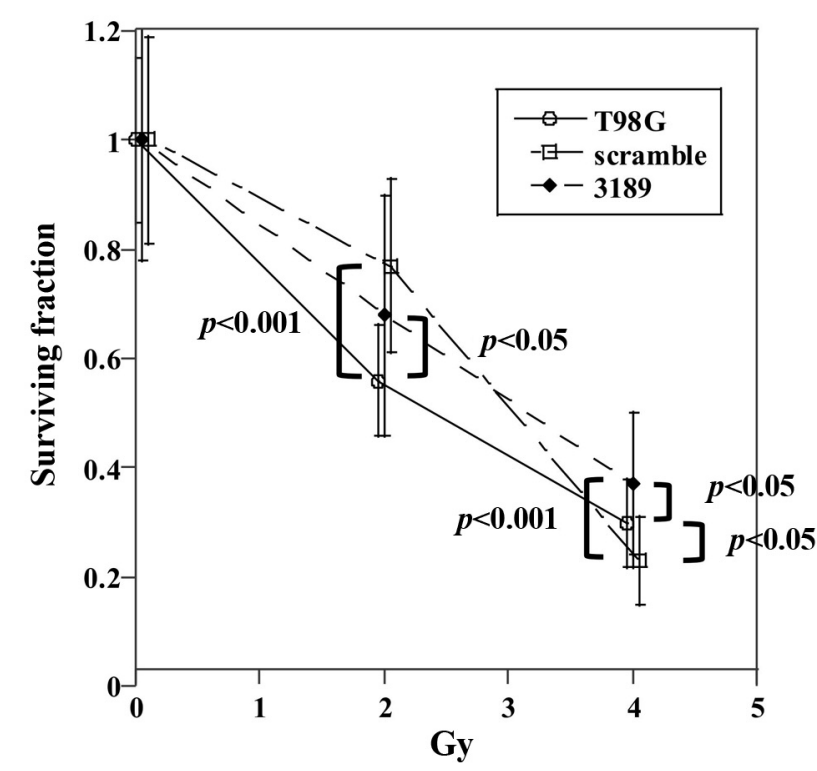

Figure 2. Effect of irradiation on colony forming capacity of the wild type, scramble short hairpin RNA (shRNA)-transfected, and nicotinamide phosphoribosyltransferase (NAMPT) shRNA-transfected $T 98 G$ cells. The effect of radiation was examined by evaluating the colony forming capacity. The wildtype, scramble shRNA-transfected, and NAMPT shRNA-transfected T98G cells were irradiated with 0, 2, or 4 Gy of X-ray and seeded in 6-well sterile plates in triplicates.

scramble shRNA-transfected, and NAMPT shRNAtransfected cells were $20.8,23.8$, and 28.6 h, respectively.

Effect of TMZ treatment and X-ray irradiation on NAMPT shRNA-transfected cells. The DNA is damaged upon administration of TMZ or exposure to radiation. During the DNA damage repair, $\mathrm{NAD}(+)$ is consumed with the activation of poly (ADP-ribose) polymerase 1 (PARP-1). Therefore, treatment with $\mathrm{NAD}(+)$ synthesis inhibitor may enhance the sensitivity of tumor cells toward TMZ or radiation (13). We investigated the sensitivity to TMZ of NAMPT shRNA-transfected cells using cytotoxicity tests. As a result, the sensitivity to TMZ of the NAMPT shRNAtransfected cells was not found to be significantly different from that of the wildtype or scramble shRNA-transfected cells. The $\mathrm{IC}_{50}$ values of TMZ against the wildtype, scramble shRNA-transfected, and NAMPT shRNA-transfected cells were 454, 600, and $546 \mu \mathrm{M}$, respectively.

Additionally, we investigated the effect of X-ray radiation on the cell self-renewal ability of the NAMPT shRNAtransfected cells by colony formation assay. The sensitivity to 2 Gy X-ray radiation of the NAMPT shRNA-transfected cells was not significantly different from that of the scramble shRNA-transfected cells. However, the sensitivity to $4 \mathrm{~Gy}$ $\mathrm{X}$-ray radiation of the NAMPT shRNA-transfected cells was

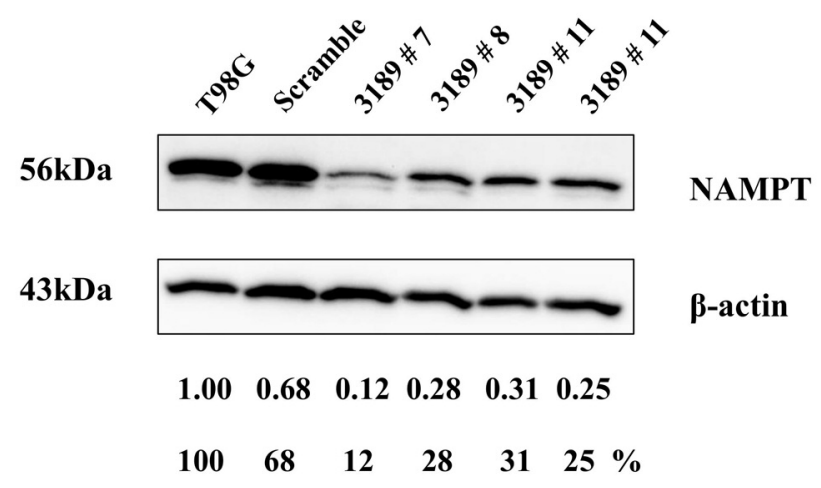

Figure 3. Nicotinamide phosphoribosyltransferase (NAMPT) expression levels increased in the NAMPT short hairpin (shRNA)-transfected cells depending on the cell passage level. The western blotting analysis of NAMPT expression at different passage levels of the wildtype, scramble shRNA-transfected, and NAMPT shRNA-transfected T98G cells is shown. The NAMPT expression level markedly increased with the increase in cell passage number. "passage number.

significantly lower $(p<0.001)$ than that of the scramble shRNA-transfected cells (Figure 2).

Effect of cell passaging on shRNA-mediated NAMPT downregulation. During the experiments, we observed that the shRNA-mediated down-regulation of NAMPT expression was rapidly restored over time. Therefore, we investigated the expression of NAMPT in the NAMPT shRNA-transfected cells at different cell passage levels. The expression level of NAMPT increased with the cell passage number (Figure 3).

Quantification of NADt, NADH, NAD(+), NADPt, NADPH, and NADP(+) levels in the NAMPT shRNA-transfected cells. We quantified the levels of NADt $[\mathrm{NAD}(+)$ and NADH], $\mathrm{NADH}$, and $\mathrm{NAD}(+)$ in the NAMPT shRNA-transfected cells exhibiting the lowest expression level of NAMPT (exhibiting $12 \%$ of the NAMPT expression level observed in the wildtype cells). The NAMPT shRNA-transfected cells exhibited significantly decreased levels of NADt and $\mathrm{NAD}(+) \quad(p<0.001$ and $p<0.01)$ when compared to the scramble shRNA-transfected cells. However the levels of NADt and NAD $(+)$ in the NAMPT shRNA-transfected cells were similar to those of wildtype cells. The NAMPT shRNA-transfected cells exhibited significantly downregulated NADH levels when compared to the wildtype $(p<0.0001)$ and scramble shRNA-transfected cells $(p<0.0001)$. Additionally, the NAMPT shRNA-transfected cells exhibited a significantly increased NAD/NADH ratio compared to the wildtype $(p<0.001)$ or scramble shRNAtransfected cells. $(p<0.001)$ (Figure 4$)$. 

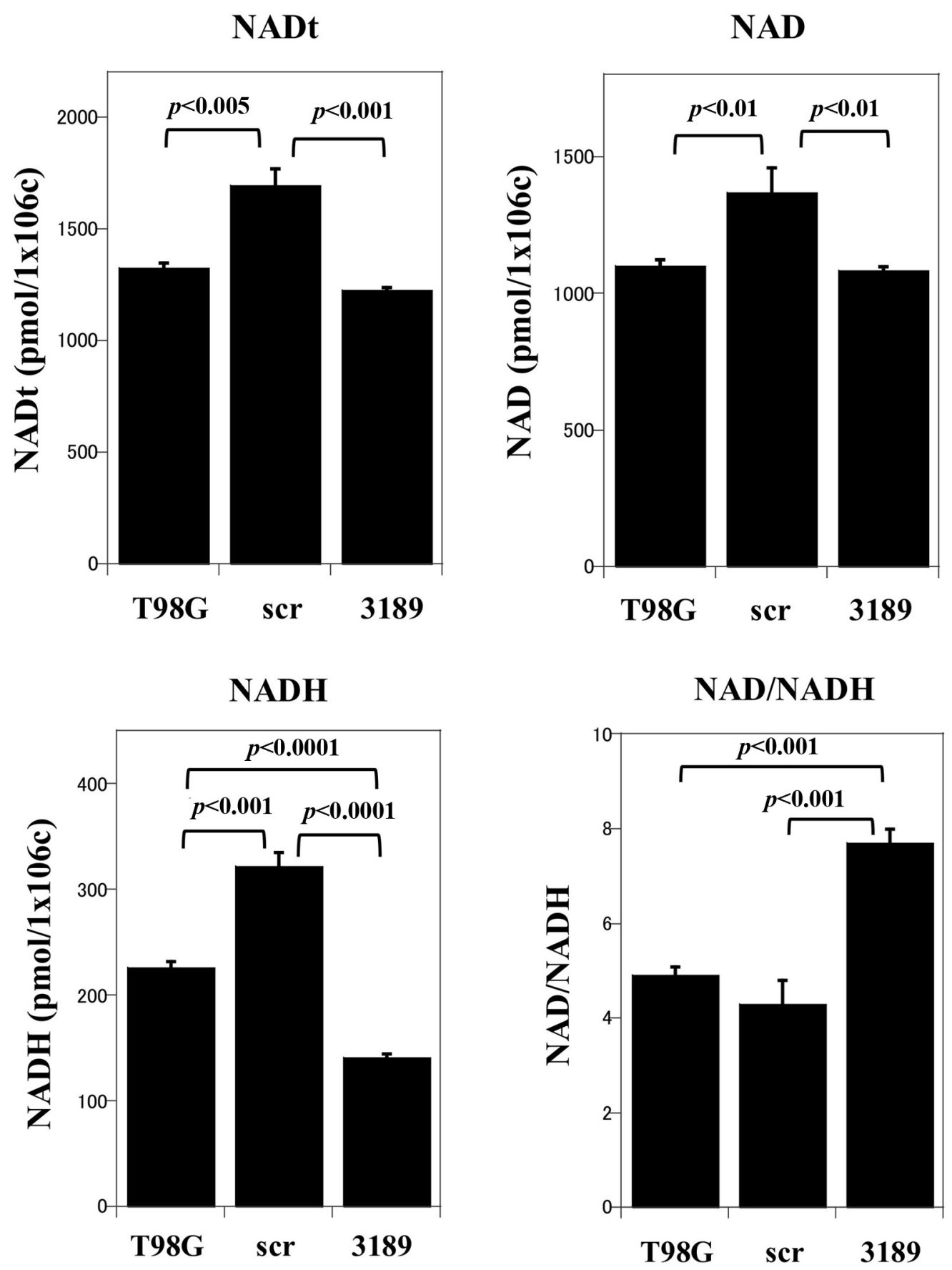

Figure 4. Quantification of the intracellular $N A D t[N A D H$ and $N A D(+)], N A D H$, and $N A D(+)$ levels in wildtype, scramble short hairpin RNA (shRNA)-transfected, and nicotinamide phosphoribosyltransferase (NAMPT) shRNA-transfected T98G cells. The intracellular NADt, NADH, NAD(+) levels were determined using the NAD/NADH Quantification Colorimetric Kit. We used the NAMPT shRNA-transfected cells exhibiting 12\% of the NAMPT expression level observed in the wildtype cells for analysis.

The NAMPT shRNA-transfected cells exhibited decreased levels of NADPt when compared to the wildtype or scramble shRNA-transfected cells. Additionally the NADPH levels in the NAMPT shRNA-transfected cells were significantly lower than those in the wildtype $(p<0.01)$ and scramble shRNA-transfected $(p<0.005)$ cells. The NADP levels were not significantly different between the wildtype, NAMPT shRNA-transfected, and scramble shRNA-transfected cells. 

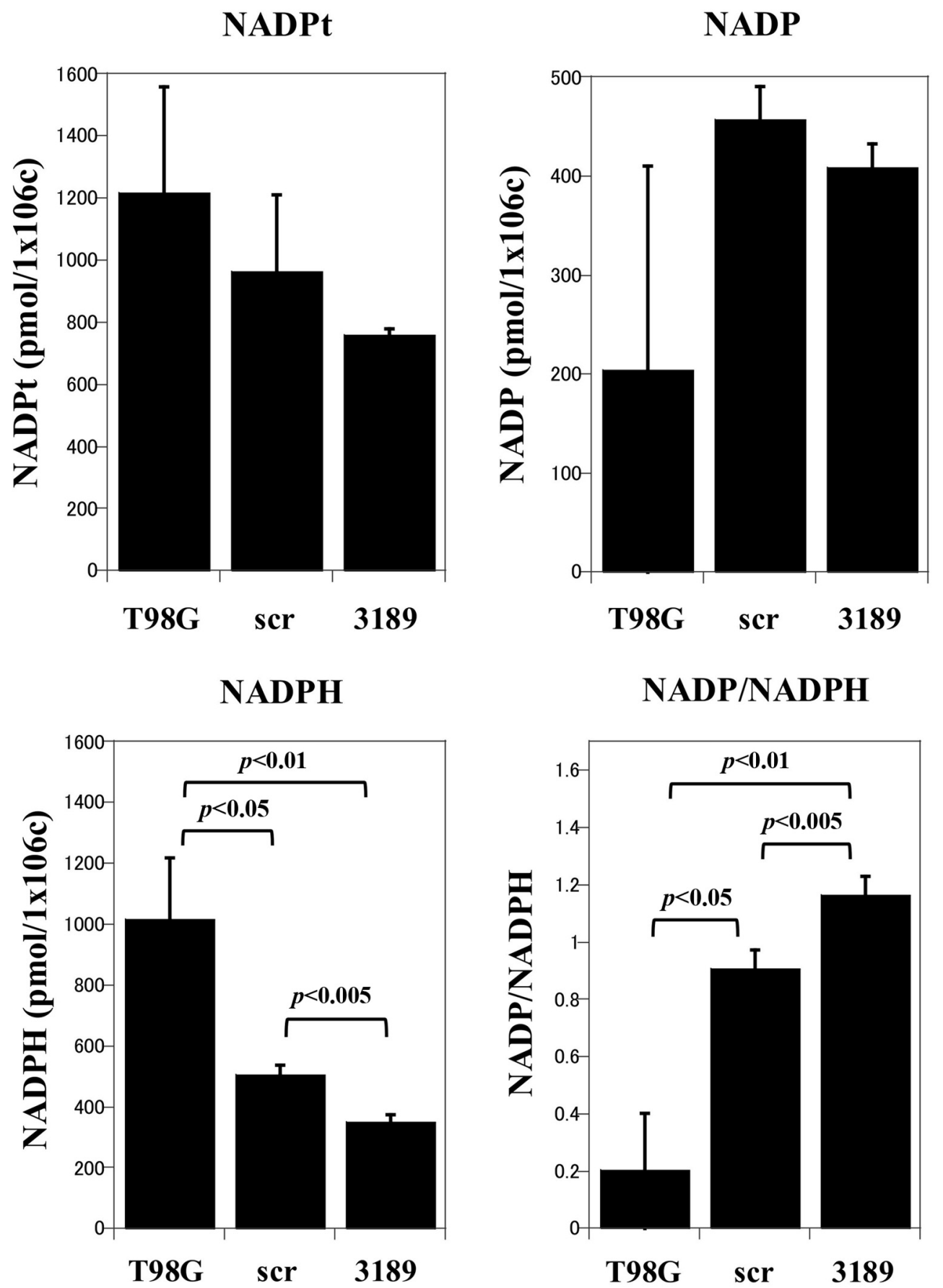

Figure 5. Quantification of intracellular NADPt [NADPH and NADP(+)], NADPH, and NADP(+) levels in wildtype, scramble short hairpin RNA (shRNA)-transfected, and nicotinamide phosphoribosyltransferase (NAMPT) shRNA-transfected T98G cells. The intracellular NADPt, NADPH, $N A D P(+)$ levels were measured using the NADP/NADPH Assay Kit-WST. We used the NAMPT shRNA-transfected cells exhibiting $12 \%$ of the NAMPT expression level observed in the wildtype cells for analysis.

However, the NAMPT shRNA-transfected cells exhibited a significantly higher NADP/NADPH ratio than the scramble shRNA-transfected $(p<0.005)$ and wildtype $(p<0.01)$ cells (Figure 5).
Investigation of activation of different $N A D(+)$ synthesis pathways. In mammals, $\mathrm{NAD}(+)$ is synthesized from NAM, NA, NR, and tryptophan. The rate-limiting step in the pathways involving NA and NR are NAPRT and NRK, 


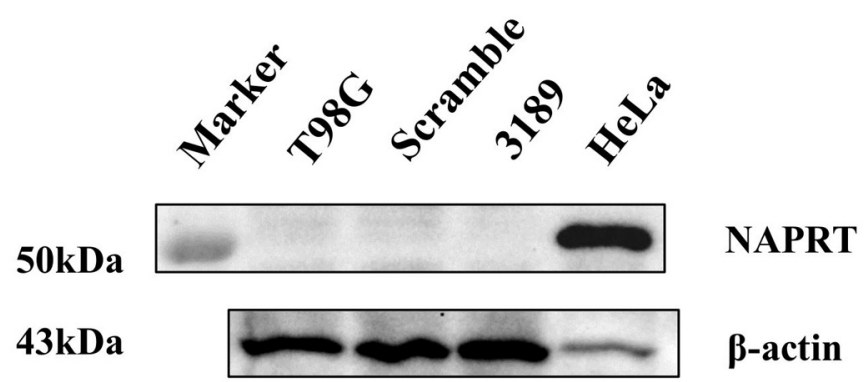

$56 \mathrm{kDa}$

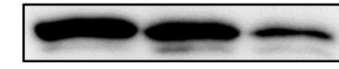

NAMPT

43kDa

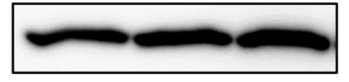

1.00

$0.60 \quad 0.16$

\section{$\beta$-actin}

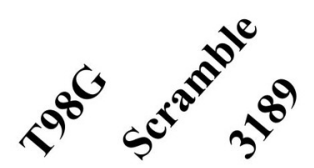

$31 \mathrm{kDa}$

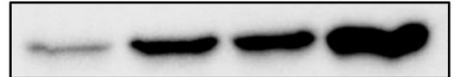

QPRT

43kDa

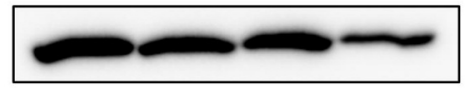

$\beta$-actin $\begin{array}{llll}1.0 & 8.1 & 8.0 & 65.4\end{array}$

$56 \mathrm{kDa}$

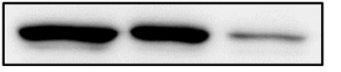

NAMPT

43kDa

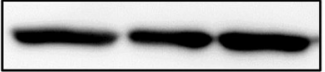

$\beta$-actin

$\begin{array}{llll}1.00 & 0.88 & 0.08\end{array}$

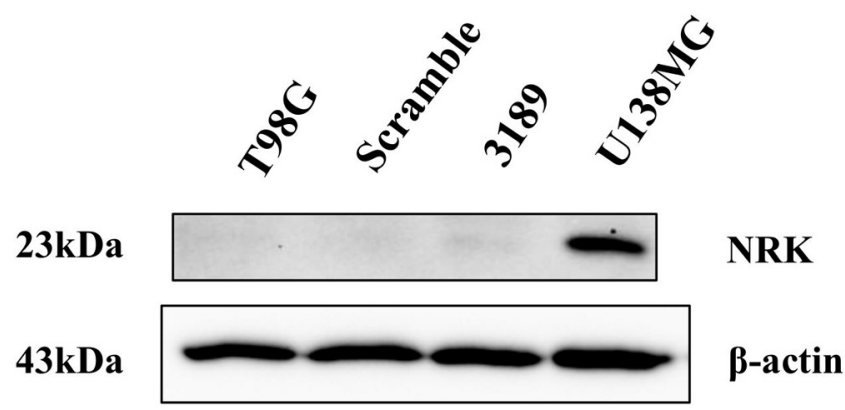

$56 \mathrm{kDa}$

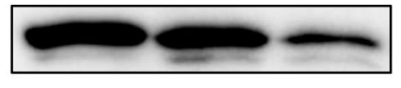

NAMPT

43kDa

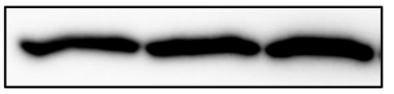

$\beta$-actin

\section{$\begin{array}{lll}1.00 & 0.60 & 0.16\end{array}$}

Figure 6. Western blot analysis of the expression levels of nicotinic acid phosphoribosyltransferase (NAPRT), nicotinamide riboside kinase (NRK), and quinolinic acid phosphoribosyltransferase (QPRT) in wildtype, scramble short hairpin (shRNA)-transfected, and nicotinamide phosphoribosyltransferase (NAMPT) shRNA-transfected T98G cells. The expression of NAPRT and NRK was not detected in the NAMPT shRNAtransfected cells that exhibit 16\% of the NAMPT expression observed in the wildtype cells. The QPRT expression level in the NAMPT shRNAtransfected cells that exhibit $8 \%$ of the NAMPT expression level observed in the wildtype cells was 8-fold higher than that in the wildtype cells. The expression level of QPRT was similar between the NAMPT shRNA-transfected cells and scramble shRNA-transfected cells.

respectively. The key enzyme of the pathway from tryptophan is QPRT $(3,4)$.

We investigated the possibility that the effect of NAMPT down-regulation on the TMZ/radiation sensitivity was not evident, as $\mathrm{NAD}(+)$ is produced via these pathways except for NAMPT-mediated pathway. The immunoblotting analysis was used to determine the expression levels of key enzymes involved in each pathway to infer the presence or absence of $\mathrm{NAD}(+)$ production by different pathways (Figure 6).

Immunoblotting analysis revealed that wildtype $\mathrm{T} 98 \mathrm{G}$ cells exhibited a mild expression of QPRT and almost no NAPRT and NRK. Additionally, expression of NAPRT or NRK was not detected in the NAMPT shRNA-transfected cells exhibiting $16 \%$ of the NAMPT expression level observed in 

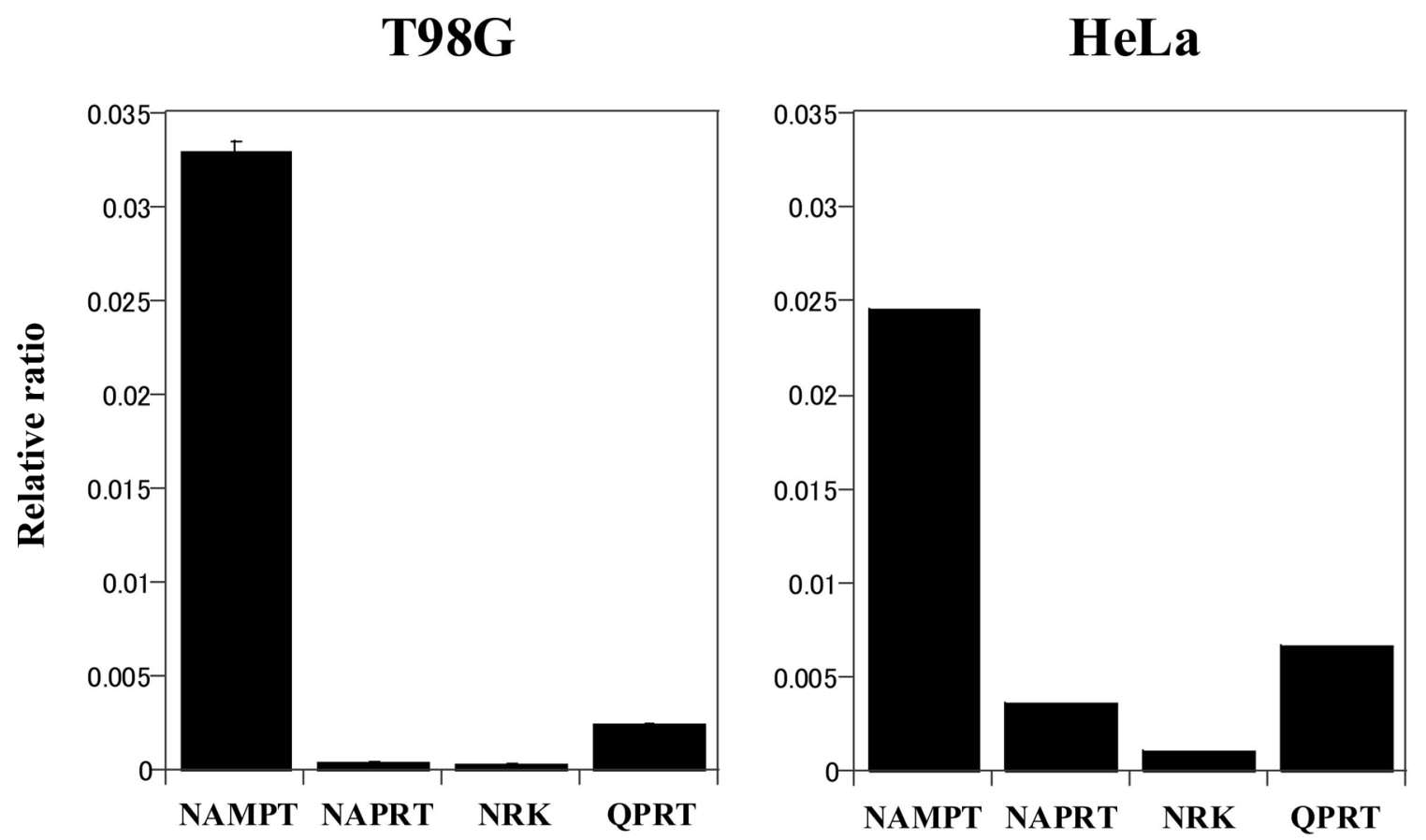

Figure 7. mRNA expression levels of NAMPT, nicotinic acid phosphoribosyltransferase (NAPRT), nicotinamide riboside kinase 1 (NRK 1), and quinolinic acid phosphoribosyltransferase (QPRT) in the T98G and HeLa cell lines. The mRNA levels were determined by semi-quantitative PCR. In the T98G cells, the mRNA levels of NAMPT were approximately 100-fold higher than those of NAPRT or NRK and approximately 14-fold higher than those of QPRT.

the wildtype cells. The NAMPT shRNA-transfected cells, which exhibit $8 \%$ of the NAMPT expression level observed in wildtype cells, exhibited 8-fold higher QPRT expression than the wildtype cells and similar QPRT expression level as the scramble shRNA-transfected cells.

The mRNA levels of NAMPT, NAPRT, NRK, and QPRT in the T98G and HeLa cells were comparatively evaluated to infer the production of $\mathrm{NAD}(+)$ via alternative pathways (other than NAMPT-mediated $\mathrm{NAD}(+)$ synthesis pathway) in the T98G glioma cells. The mRNA levels of NAMPT were approximately 100-fold higher than those of NAPRT or NRK and approximately 14-fold higher than those of QPRT (Figure 7).

\section{Discussion}

We generated a NAMPT down-regulated glioma cell line to evaluate the potential of shRNA-mediated NAMPT downregulation as adjuvant therapy for treating invasive glioma. The NAMPT shRNA-transfected cells exhibited down-regulated NAMPT expression and decreased cell growth. However, the TMZ sensitivity of the NAMPT shRNA-transfected cells was similar to that of the scramble shRNA-transfected cells. Additionally, irradiating the NAMPT shRNA-transfected cells with X-ray did not affect their colony formation capability.

The intracellular NADt levels in the NAMPT shRNAtransfected cells exhibiting $12 \%$ of the NAMPT expression level observed in wildtype cells were lower than those in the scramble shRNA-transfected cells. However, there was no significant difference in the NADt levels between the NAMPT shRNA-transfected and wildtype cells. This indicated that suppressing NAMPT expression in approximately $10 \%$ of the wildtype T98G glioma cells, is insufficient to reduce the NADt level, or $\mathrm{NAD}(+)$ was produced via alternative pathways.

Several studies indicated that these alternative NAD $(+)$ biosynthetic pathways must be targeted for NAMPT-based anti-cancer therapy. Some studies have reported that QPRT up-regulation is associated with conferring the tumor cells with resistance against NAMPT SMIs $(3,19)$. NAMPT inhibitors have minimal clinical effects against cancer cells in which $\mathrm{NAD}(+)$ is synthesized by NAPRT $(6,9,20-22)$ or NAMPT and NRK play the primary role in extracellular NAD(+) or NMN utilization (4) (5).

In this study, we investigated the expression of key enzymes, other than NAMPT, that are involved in the NAD(+) synthetic pathways in T98G glioma cells. The T98G glioma cells exhibited QPRT expression, but not NRK expression. Several splice variants of NAPRT proteins with an estimated molecular weight of $57.6,56.1$, and $52.2 \mathrm{kDa}$ were not confirmed in the study. Therefore, the expression level of NAPRT should be minimal if any in the T98G glioma cells that is consistent to RT-PCR results. We also investigated the induction of expression of NAPRT, NRK 
and QPRT in the NAMPT shRNA-transfected cells. The expression of NAPRT or NRK was not detected in the NAMPT shRNA-transfected cells exhibiting $12 \%$ of the NAMPT expression level observed in the wildtype cells.

The expression level of QPRT in the NAMPT shRNAtransfected cells, that exhibited $8 \%$ of the NAMPT expression compared to wildtype cells, was about 8 times that in the wildtype cells. This increase might suggest $\mathrm{NAD}(+)$ production from tryptophan. This may be the reason for the NAMPT shRNA-transfected cells exhibiting similar NADt level and TMZ/radiation sensitivity as wildtype cells. However, the expression levels of QPRT were similar between the scramble shRNA-transfected and NAMPT shRNA-transfected cells. This suggested that down-regulation of NAMPT did not induce enhanced QPRT protein expression. The agents that induce oxidative stress, such as TMZ or radiation exposure are known to up-regulate QPRT expression in the glioma cells (23), which suggests that the stimuli of retrovirus vector transfection can promote QPRT expression.

Similar to NAMPT expression, QPRT expression is dependent on tumor grade. One study reported that enhanced QPRT expression can increase the resistance against chemotherapy-mediated oxidative stress and that the high QPRT expression observed in the recurrent glioblastoma after chemotherapy is correlated with a poor prognosis (23). This study suggested that similar to the NAMPT-catalyzed $\mathrm{NAD}(+)$ production, QPRT-catalyzed $\mathrm{NAD}(+)$ production can contribute to the malignant phenotype of glioma cells. The mRNA expression analysis indicated that the contribution of NAMPT toward NAD $(+)$ synthesis is higher than that of QPRT in the T98G glioma cells. However, the QPRTcatalyzed synthesis of NAD(+) in the NAMPT shRNAtransfected cells may contribute to resistance against TMZ or radiation exposure, particularly when NAMPT expression level is low.

Since both NAMPT and QPRT have an effect on malignant phenotype through $\mathrm{NAD}(+)$ production, the effect might be similar on the biology of the glioma cells, however, Sahm et al. demonstrated that QPRT produces $\mathrm{NAD}(+)$ by utilizing quinolinic acid derived from microglia which infiltrate to glioblastoma in vivo (23). Therefore, QPRT may have an effect on malignancy depends on availability of quinolinic acid as a substrate.

Both $\mathrm{NAD}(+)$ and $\mathrm{NMN}$ are present in plasma. The function of NRK, which phosphorylates the absorbed NR into NMN, is essential for the utilization of the extracellular $\mathrm{NAD}(+)$ precursor $(4,5)$. NRK expression was rarely detected in the NAMPT shRNA-transfected cells, which indicated that the extracellular $\mathrm{NAD}(+)$ precursor derived NR may not be used for NAD(+) synthesis in the NAMPT shRNA-transfected cells.

The levels of NADH or NADPH in the NAMPT shRNAtransfected cells were significantly lower than those of the wildtype and scramble shRNA-transfected cells. The NAMPT shRNA-transfected cells exhibited higher NAD/NADH and NADP/NADPH ratios than the wildtype and scramble shRNA-transfected cells. Cerna et al. reported that inhibiting $\mathrm{NAD}(+)$ synthesis in the tumor cells by NAMPT inhibitor treatment increases the reactive oxygen species (ROS) level, which subsequently induces apoptosis, by decreasing the levels of NADH, NADP(+), and NADPH. These substances function as reducing agents (20). In addition Feng et al. indicated that low doses of NAMPT inhibitors alone did not exhibit cytotoxicity, yet increased sensitivity to TMZ by both increasing ROS and activating JNK signal pathways (8). Wang et al. reported that NAMPT knockdown significantly decreases the mRNA levels of catalase (CAT) and manganese superoxide dismutase (SOD) in prostatic carcinoma cells and that NAMPT inhibitors and NAMPT knockdown significantly decreases the expression of ROXO3a, which is the direct transcriptional activator of the CAT and SOD promoter (24).

In this study, the NAMPT shRNA-transfected cells did not decrease NADt or NADPt expression. However, the increase in the NAD/NADH ratio and NADP/NADPH ratio may be caused due to reduced NADH and NADPH consumption to protect the cells against ROS-induced damages.

Wang and Guo reported that lentiviral shNAMPTtransfected cells inhibited cell growth, colony formation, migration, apoptosis, and invasive capacity, as well as decreased in vivo growth of xenografted cancer cells $(24,25)$.

In this experiment, the NAMPT shRNA-transfected cells did not exhibit enhanced TMZ/radiation sensitivity, although the growth of shRNA-transfected cells was delayed. This may be because of the insufficient decrease of NADt caused due to QPRT-catalyzed NAD (+) synthesis or due to the selection mechanism that selects the cells exhibiting enhanced NAMPT expression. Wang et al. reported that the intracellular NAD level in the shNAMPTtransfected cells decreased by approximately $20 \%$ within five days after transduction. They investigated the changes in cell phenotypes immediately after transduction. Some of these phenotypes were evaluated by concentrating the lentiviral shNAMPT vector-transfected cells by sorting. Moreover Guo et al. also generated cell lines by infecting the NAMPT shRNA-expressing lentiviral vector to investigate the changes in cell phenotype. Their NAMPT shRNA-transfected cells exhibited more than $90 \%$ infection efficiency after $72 \mathrm{~h}$ of infection. The discrepancy in the results of their study and ours may be attributed to the differences in cell lines used or the puromycin selection. The most important finding in this study was that the protein expression levels of NAMPT in the NAMPT shRNA-transfected cells, which exhibit $8 \%$ of the NAMPT expression level observed in the wildtype cells, increased with the increase in the cell passage levels. 
Several studies have reported that the enhanced NAMPT protein expression is the mechanism underlying resistance against NAMPT SMIs, such as GMX1778 or APO866 (3, $26,27)$. The $\mathrm{IC}_{50}$ values of various cells against NAMPT inhibitors are reported to be correlated with the mRNA levels of NAMPT (22).

NAMPT shRNA-transfected cells, which exhibit $18 \%$ of NAMPT expression level observed in the wildtype cells, exhibited delayed cell growth. Additionally, the NAMPT expression level increases rapidly with the increase in the cell passage numbers. The fast-growing cells may be selected during the propagation of the sub-cultured cells. This indicated that the effect of shRNA-mediated NAMPT inhibition decreased over time. Therefore, alternative methods of NAMPT suppression or exploration of more applicable molecular targets might be important.

\section{Conclusion}

We demonstrated that shRNA-mediated NAMPT downregulation inhibit the cell growth. However, as passage of cells increased, the effect of inhibition of NAMPT decreased. In addition, the method did not decrease the NADt to a sufficient level to increase TMZ/radiation sensitivity. Alternative methods of NAMPT suppression or exploration of more applicable molecular targets need to be explored.

\section{Conflicts of Interest}

The Authors have no conflicts of interest to declare.

\section{Authors' Contributions}

YM designed the study. MK and KI conducted the experiments and MK interpreted the results. MK wrote the paper. YM supervised the study.

\section{Acknowledgements}

The Authors acknowledge Dr. Kouki Fujioka, Dr. Yumi Kanegae and Dr. Satomi Yogosawa of the Jikei University School of Medicine for helpful discussion. We also thank Mt. Hidetoshi Izumiya, Ms. Keiko Tomaru and Mayumi Nomura for their expert technical assistance.

\section{References}

1 Goellner EM, Grimme B, Brown AR, Lin YC, Wang XH, Sugrue KF, Mitchell L, Trivedi RN, Tang JB and Sobol RW: Overcoming temozolomide resistance in glioblastoma via dual inhibition of nad+ biosynthesis and base excision repair. Cancer Res 71(6): 2308-2317, 2011. PMID: 21406402. DOI: 10.1158/0008-5472.CAN-10-3213

2 Garten A, Schuster S, Penke M, Gorski T, de Giorgis T and Kiess W: Physiological and pathophysiological roles of nampt and nad metabolism. Nat Rev Endocrinol 11(9): 535-546, 2015.
PMID: 26215259. DOI: 10.1038/nrendo.2015.117

3 Guo J, Lam LT, Longenecker KL, Bui MH, Idler KB, Glaser KB, Wilsbacher JL, Tse C, Pappano WN and Huang TH: Identification of novel resistance mechanisms to nampt inhibition via the de novo nad(+) biosynthesis pathway and nampt mutation. Biochem Biophys Res Commun 491(3): 681686, 2017. PMID: 28756225. DOI: 10.1016/j.bbrc.2017.07.143

4 Grozio A, Sociali G, Sturla L, Caffa I, Soncini D, Salis A, Raffaelli N, De Flora A, Nencioni A and Bruzzone S: Cd73 protein as a source of extracellular precursors for sustained nad+ biosynthesis in fk866-treated tumor cells. J Biol Chem 288(36): 25938-25949, 2013. PMID: 23880765. DOI: 10.1074/jbc.M113.470435

5 Ratajczak J, Joffraud M, Trammell SA, Ras R, Canela N, Boutant M, Kulkarni SS, Rodrigues M, Redpath P, Migaud ME, Auwerx J, Yanes O, Brenner C and Canto C: Nrk1 controls nicotinamide mononucleotide and nicotinamide riboside metabolism in mammalian cells. Nat Commun 7(13103, 2016. PMID: 27725675. DOI: 10.1038/ncomms 13103

6 Piacente F, Caffa I, Ravera S, Sociali G, Passalacqua M, Vellone VG, Becherini P, Reverberi D, Monacelli F, Ballestrero A, Odetti P, Cagnetta A, Cea M, Nahimana A, Duchosal M, Bruzzone S and Nencioni A: Nicotinic acid phosphoribosyltransferase regulates cancer cell metabolism, susceptibility to nampt inhibitors, and DNA repair. Cancer Res 77(14): 3857-3869, 2017. PMID: 28507103. DOI: 10.1158/0008-5472.CAN-16-3079

7 Lucena-Cacace A, Otero-Albiol D, Jimenez-Garcia MP, PeinadoSerrano J and Carnero A: Nampt overexpression induces cancer stemness and defines a novel tumor signature for glioma prognosis. Oncotarget 8(59): 99514-99530, 2017. PMID: 29245920. DOI: 10.18632 /oncotarget.20577

8 Feng J, Yan PF, Zhao HY, Zhang FC, Zhao WH and Feng M: Inhibitor of nicotinamide phosphoribosyltransferase sensitizes glioblastoma cells to temozolomide via activating ros/jnk signaling pathway. Biomed Res Int 2016: 1450843, 2016. PMID: 28097126. DOI: 10.1155/2016/1450843

9 Duarte-Pereira S, Pereira-Castro I, Silva SS, Correia MG, Neto C, Costa LT, Amorim A and Silva RM: Extensive regulation of nicotinate phosphoribosyltransferase (naprt) expression in human tissues and tumors. Oncotarget 7(2): 1973-1983, 2015. PMID: 26675378. DOI: 10.18632 /oncotarget.6538

10 Tateishi K, Higuchi F, Miller JJ, Koerner MVA, Lelic N, Shankar GM, Tanaka S, Fisher DE, Batchelor TT, Iafrate AJ, Wakimoto H, Chi AS and Cahill DP: The alkylating chemotherapeutic temozolomide induces metabolic stress in idh1-mutant cancers and potentiates nad(+) depletion-mediated cytotoxicity. Cancer Res 77(15): 4102-4115, 2017. PMID: 28625978. DOI: 10.1158/0008-5472.CAN-16-2263

11 Kato H, Ito E, Shi W, Alajez NM, Yue S, Lee C, Chan N, Bhogal N, Coackley CL, Vines D, Green D, Waldron J, Gullane P, Bristow R and Liu FF: Efficacy of combining gmx 1777 with radiation therapy for human head and neck carcinoma. Clin Cancer Res 16(3): 898-911, 2010. PMID: 20103674. DOI: 10.1158/1078-0432.ccr-09-1945

12 Zerp SF, Vens C, Floot B, Verheij M and van Triest B: Nad(+) depletion by apo866 in combination with radiation in a prostate cancer model, results from an in vitro and in vivo study. Radiother Oncol 110(2): 348-354, 2014. PMID: 24412016. DOI: 10.1016/j.radonc.2013.10.039

13 Elf AK, Bernhardt P, Hofving T, Arvidsson Y, Forssell-Aronsson E, Wangberg B, Nilsson O and Johanson V: Nampt inhibitor 
gmx1778 enhances the efficacy of 177lu-dotatate treatment of neuroendocrine tumors. J Nucl Med 58(2): 288-292, 2017. PMID: 27688470. DOI: 10.2967/jnumed.116.177584

14 Manome Y, Kunieda, T, Wen PW, Koga T, Kufe DW and Ohno $\mathrm{T}$ : Transgene expression in malignant glioma using a replicationdefective adenoviral vector containing the egr-1 promoter: Activation by ionizing radiation or uptake of radioactive iododeoxyuridine. Hum Gene Ther 9: 1409-1417, 1998. PMID: 9681412.

15 Namba H, Kawaji $H$ and Yamasaki T: Use of genetically engineered stem cells for glioma therapy. Oncol Lett 11(1): 915, 2016. PMID: 26870161. DOI: 10.3892/ol.2015.3860

16 van Horssen R, Willemse M, Haeger A, Attanasio F, Guneri T, Schwab A, Stock CM, Buccione R, Fransen JA and Wieringa B: Intracellular nad(h) levels control motility and invasion of glioma cells. Cell Mol Life Sci 70(12): 2175-2190, 2013. PMID: 23307072. DOI: 10.1007/s00018-012-1249-1

17 Manome Y, Abe M, Hagen MF, Fine HA, Kufe DW: Enhancer sequences of the $d f 3$ gene regulate expression of the herpes simplex virus thymidine kinase gene and confer sensitivity of human breast cancer cells to ganciclovir. Cancer Res Treat 54: 5408-5413, 1994. PMID: 7923173.

18 Wang Z, Gao Y, Zhang C, Hu H, Guo D, Xu Y, Xu Q, Zhang W, Deng S, Lv P, Yang Y, Ding Y, Li Q, Weng C, Chen X, Gong S, Chen H, Niu J and Tang H: Quinolinate phosphoribosyltransferase is an antiviral host factor against hepatitis c virus infection. Sci Rep 7(1): 5876, 2017. PMID: 28724915. DOI: 10.1038/s41598-017-06254-4

19 Thongon N, Zucal C, D’Agostino VG, Tebaldi T, Ravera S, Zamporlini F, Piacente F, Moschoi R, Raffaelli N, Quattrone A, Nencioni A, Peyron JF and Provenzani A: Cancer cell metabolic plasticity allows resistance to nampt inhibition but invariably induces dependence on ldha. Cancer Metab 6: 1, 2018. PMID: 29541451. DOI: 10.1186/s40170-018-0174-7

20 Cerna D, Li H, Flaherty S, Takebe N, Coleman CN and Yoo SS: Inhibition of nicotinamide phosphoribosyltransferase (nampt) activity by small molecule gmx 1778 regulates reactive oxygen species (ros)-mediated cytotoxicity in a p53- and nicotinic acid phosphoribosyltransferase1 (naprt1)-dependent manner. J Biol Chem 287(26): 22408-22417, 2012. PMID: 22570471. DOI: $10.1074 / \mathrm{jbc} . \mathrm{M} 112.357301$

21 Olesen UH, Thougaard AV, Jensen PB and Sehested M: A preclinical study on the rescue of normal tissue by nicotinic acid in high-dose treatment with apo866, a specific nicotinamide phosphoribosyltransferase inhibitor. Mol Cancer Ther 9(6): 1609-1617, 2010. PMID: 20515945. DOI: 10.1158/15357163.MCT-09-1130
22 Watson M, Roulston A, Belec L, Billot X, Marcellus R, Bedard D, Bernier C, Branchaud S, Chan H, Dairi K, Gilbert K, Goulet D, Gratton MO, Isakau H, Jang A, Khadir A, Koch E, Lavoie M, Lawless M, Nguyen M, Paquette D, Turcotte E, Berger A, Mitchell M, Shore GC and Beauparlant P: The small molecule gmx1778 is a potent inhibitor of nad+ biosynthesis: Strategy for enhanced therapy in nicotinic acid phosphoribosyltransferase 1deficient tumors. Mol Cell Biol 29(21): 5872-5888, 2009. PMID: 19703994. DOI: 10.1128/MCB.00112-09

23 Sahm F, Oezen I, Opitz CA, Radlwimmer B, von Deimling A, Ahrendt T, Adams S, Bode HB, Guillemin GJ, Wick W and Platten M: The endogenous tryptophan metabolite and nad+ precursor quinolinic acid confers resistance of gliomas to oxidative stress. Cancer Res 73(11): 3225-3234, 2013. PMID: 23548271. DOI: 10.1158/0008-5472.CAN-12-3831

24 Wang B, Hasan MK, Alvarado E, Yuan H, Wu H and Chen WY: Nampt overexpression in prostate cancer and its contribution to tumor cell survival and stress response. Oncogene 30(8): 907921, 2011. PMID: 20956937. DOI: 10.1038/onc.2010.468

25 Guo Q, Han N, Shi L, Yang L, Zhang X, Zhou Y, Yu S and Zhang M: Nampt: A potential prognostic and therapeutic biomarker in patients with glioblastoma. Oncol Rep 42(3): 963972, 2019. PMID: 31322259. DOI: 10.3892/or.2019.7227

26 Wang W, Elkins K, Oh A, Ho YC, Wu J, Li H, Xiao Y, Kwong M, Coons M, Brillantes B, Cheng E, Crocker L, Dragovich PS, Sampath D, Zheng X, Bair KW, O'Brien T and Belmont LD: Structural basis for resistance to diverse classes of nampt inhibitors. PLoS One 9(10): e109366, 2014. PMID: 25285661. DOI: 10.1371/journal.pone.0109366

27 Olesen UH, Petersen JG, Garten A, Kiess W, Yoshino J, Imai S, Christensen MK, Fristrup P, Thougaard AV, Bjorkling F, Jensen PB, Nielsen SJ and Sehested M: Target enzyme mutations are the molecular basis for resistance towards pharmacological inhibition of nicotinamide phosphoribosyltransferase. BMC Cancer 10(677, 2010. PMID: 21144000. DOI: 10.1186/14712407-10-677

Received May 27, 2020

Revised June 15, 2020

Accepted June 22, 2020 